\title{
Fair Sharing of MAC under TCP in Wireless Ad Hoc Networks
}

\author{
Ken Tang, Mario Gerla \\ $\{k$ tang, gerla\}@cs.ucla.edu \\ Wireless Adaptive Mobility Laboratory \\ Computer Science Department \\ University of California, Los Angeles \\ Los Angeles, CA 90095 \\ http://www.cs.ucla.edu/NRL/wireless
}

\begin{abstract}
In this study we investigate the performance of TCP and MAC layer in a wireless multi-hop network. Using simulation, we provide new insights into the interactions between TCP and various MAC layer protocols, including CSMA, FAMA and 802.11. These MAC protocols were chosen because they provide an evolution of wireless medium access schemes, starting with carrier sensing (CSMA), then evolving to the utilization of RTS/CTS control frames (FAMA) and finally progressing to collision avoidance and acknowledgements (802.11). We examine these interactions in various network topologies and in a mobile environment where node movements are unpredictable. In particular, we address the issue of fair sharing of MAC with multiple TCP flows.
\end{abstract}

\section{Introduction}

The rapid advancement in portable computing platforms and wireless communication technology has led to significant interest in the design and development of protocols for instantly deployable, wireless networks often referred to as "ad hoc networks". Ad hoc networks are required in situations where a fixed communication infrastructure, wired or wireless, does not exist or has been destroyed. The applications span several different sectors of society. In the civilian environment, they can be used to interconnect workgroups moving in an urban or rural area or a campus and engaged in collaborative operation such as distributed scientific experiments and search and rescue. In the law enforcement sector, applications such as crowd control and border patrol come to mind. In the military arena, the modern communications in a battlefield theater require a very sophisticated instant infrastructure with far more complex requirements and constraints than the civilian applications [8].

In such environments, reliable data transfer and congestion control is paramount. TCP is generally used to support these features. However, as shown in [9], depending on the MAC layer being used, TCP can exhibit capture behavior that is unacceptable in these critical environments where multiple TCP flows on a single node are common. One node capturing the wireless channel while the other nodes are being locked out can result in catastrophic outcomes in the search and rescue or military operations. Thus, an important issue that we address in our study is the effect of TCP performance with multiple flows on such ad hoc networks under various MAC.

In this paper, we study the TCP/MAC layer interaction via simulation, specifically emphasizing on fair sharing of MAC under TCP. The simulation platform used is GloMoSim [12]. GloMoSim is 
a discrete event, parallel simulation environment implemented in PARSEC, PARallel Simulation Environment for Complex Systems [1]. It includes various wireless protocols in its library (radio propagation, mobility, MAC, network, transport and applications). In addition, GloMoSim provides a valuable and useful feature that facilitates different protocols at a given layer to be swapped in and out of the protocol stack and thus allows for comparison between these different protocols. Most importantly, GloMoSim permits the detailed modeling of several layers and the study of their interaction, yet preserving very good runtime efficiency and yielding manageable execution time.

The rest of the paper is organized as follows: Section 2 reports the configuration and parameters we used for our simulation. TCP over the MAC layer simulation experiments are examined in section 3. Finally, section 4 concludes the paper.

\section{Experimental Configuration and Parameters}

For our simulation experiments, we consider several topologies (Fig. 1 - Fig. 4): string, hidden terminal, ring, and grid. The arrows represent the direction of data packet transmissions. FTP with infinite backlog running on top of TCP is used for the application. We utilize static routing to route packets when mobility is not considered and use Bellman-Ford routing when mobility is introduced. Three MAC protocols are considered: CSMA, FAMA and IEEE 802.11. Radios with no capture ability are modeled with a channel bandwidth of $2 \mathrm{Mbps}$. Furthermore, the channel uses free-space with no external noise (perfect channel).

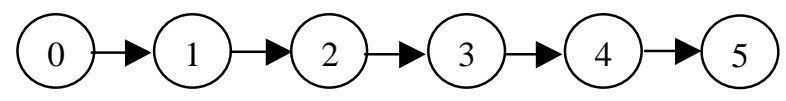

Fig. 1: String Topology

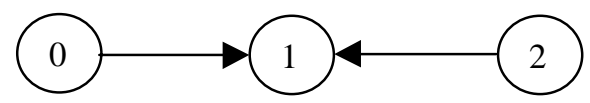

Fig. 2: Hidden Terminal Topology

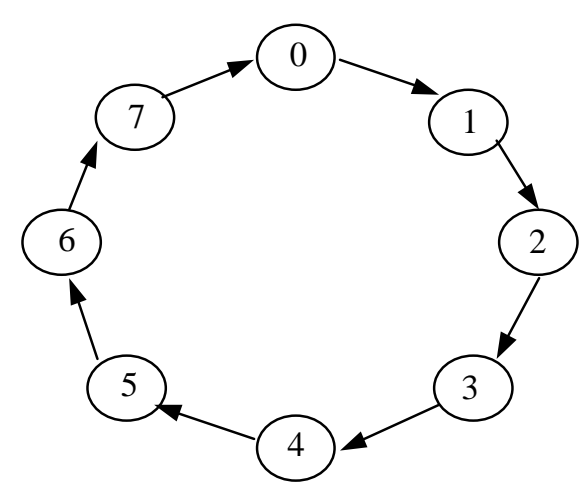

Fig. 3: Ring Topology

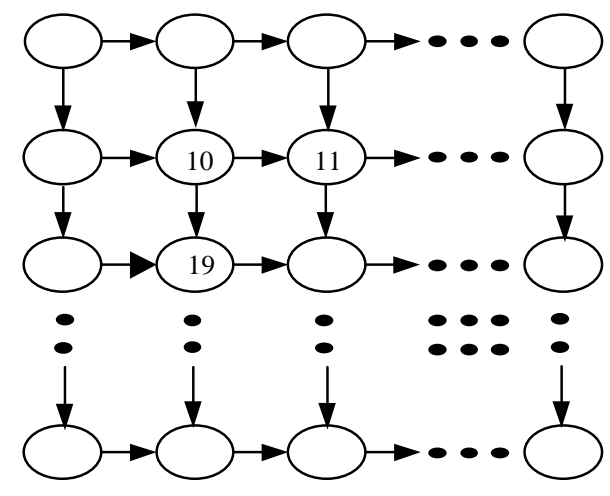

Fig. 4: Grid Topology 
CSMA, FAMA and IEEE 802.11 are chosen because they represent a progression of carrier sensing methods. CSMA (Carrier Sense Multiple Access) requires carrier sensing before transmission. If the channel is free, the packet is transmitted immediately. Otherwise, it is rescheduled after a random timeout. The major limitation of CSMA is the "hidden terminal" and "exposed terminal" problem [11]. The hidden terminal problem illustrates that collision of data packets occurs at the receiver, rather than at the sender. Even if the channel is free within the sender's range, it may not be free at the receiver. In the exposed terminal problem, although the medium is sensed busy near the transmitter, the medium may be free near the intended receiver. CSMA was used first in the Packet Radio network in the mid 1970's [10].

FAMA (Floor Acquisition Multiple Access) is an experimental MAC protocol specifically developed for the Glomo DARPA program. In addition to carrier sensing, FAMA features the RTS (Request To Send) and CTS (Clear To Send) exchange to prepare the floor for data transmission (thus avoiding hidden terminal collision in most cases) [7]. A node wanting to transmit first senses the channel. If the channel is busy, the node backs off a random amount of time and tries again. If the channel is idle, the node initiates a Request To Send (RTS) control frame, and sets a timer to await the Clear To Send (CTS) control frame from the intended receiver. Any node overhearing the RTS control frame is within range to collide with the expected incoming CTS frame, and must defer transmission for a period of time long enough to ensure the return delivery of the CTS. If the node initiating the RTS does not receive the CTS within a specified timeout interval, it assumes a collision has occurred, and initiates a backoff before attempting to retransmit. Any node overhearing the CTS control frame is within range to collide with the upcoming transmission, and is required to defer for the full duration of transmission, as specified in the control frame.

IEEE 802.11 expands on FAMA by adding link-level ACKs in addition to the RTS/CTS control frames [6]. Furthermore, 802.11 abandons the traditional CSMA in favor of CSMA/CA (Collision Avoidance). In 802.11, the Distributed Coordination Function (DCF) represents the basic access method that mobile nodes utilize to share the wireless channel. The scheme incorporates CSMA with Collision Avoidance (CSMA/CA) and acknowledgement (ACK). Optionally, the mobile nodes can make use of the virtual carrier sense mechanism that employs RTS/CTS exchange for channel reservation and fragmentation of packets in situations where the wireless channel experiences high bit error rate. CSMA/CA works as follows: a node wishing to transmit senses the channel, and if it is free for a time equal to the DCF InterFrame Space (DIFS), the node transmits. If the channel is busy, the node enters a state of collision avoidance and backs off from transmitting for a specified interval. In the collision avoidance state, the node sensing the channel busy will suspend it's backoff timer, only resuming the backoff countdown when the channel is again sensed free for a DIFS period. A typical sequence of exchange in 802.11 using the virtual carrier sensing mechanism involves the source node first sensing the channel using CSMA/CA. After CSMA/CA is executed, the source node transmits a RTS, followed by the destination node responding with a CTS, then with the source node sending the data frame and ending with the destination node confirming with an ACK to the source node.

Each node has a 25-packet MAC layer buffer pool. Scheduling of packet transmissions is FIFO. FAMA is implemented with a less aggressive yield time than in [9]. Yield time is defined as the amount of time the sender backs off before sending another data frame after transmitting a data frame. Yield time also refers to the amount of time the receiver backs off before sending a frame after receiving a data frame. 802.11 employs the DCF access method with virtual carrier sense. The TCP simulation model is an accurate replica of the TCP code running in the Internet hosts today. The TCP simulation code was generated from FreeBSD 2.2.4 code. In particular, window size grows progressively until it reaches the advertised window or until packet loss is detected. In the latter cases, window size is halved (fast retransmission and fast recovery) or abruptly reduced to 1 (slow start). In our experiments, we will "force" the maximum TCP window to take a certain value by 
setting the advertised window to such value (e.g., 1460B). TCP packet length is assumed fixed at 1460B. TCP connections are started uniformly, distributed between 0 to 10 seconds. Each simulation run is executed for 200 simulated seconds except in the grid experiments where the runs last for 400 simulated seconds.

\section{TCP over MAC Layer}

Previous TCP/MAC layer simulation experiments at UCLA $[2,10]$ have uncovered two major weaknesses of the MAC layer: (a) CSMA and, to a lesser extent, FAMA suffer from the hidden terminal losses; (b) one or more stations tend to "capture" the channel in heavy load situation. The capture phenomenon is also reported in [5] and [13]. Thus, loss recovery must be provided by a layer above MAC, either the link layer or the transport layer. In [2], we considered loss recovery only at the TCP transport layer protocol, as it is the most popular solution in wireless LANs and it is the easiest to implement. We also investigated the effectiveness of TCP in removing MAC layer capture. The first set of results we obtained from these experiments was rather negative. TCP alone does not improve throughput performance in most cases. In some cases it makes it worse. For example, in multi-hop wireless paths TCP on top of CSMA and FAMA alike dramatically degrades performance when the congestion window is greater than 1 packet. TCP mitigates capture behavior in some cases, but makes it worse in others [2]. In conclusion, we realized that additional techniques (beyond mere TCP) are necessary to obtain good performance in a multi-hop wireless environment.

As previously indicated in [9], one possible technique is the use of link level ACKs. In [9], the impact of link level ACKs in the form of MACAW was studied and shown to have a positive effect on TCP throughput. In this paper, we introduce the link level ACKs of the IEEE 802.11 MAC layer standard. Once more, we argue that increasing the TCP window greater than one packet size has no beneficial impact on network performance. This applies to all three MAC protocols being studied, CSMA, FAMA and 802.11. We then report the results of TCP performance with multiple flows in a wireless ad hoc network environment under various network configurations. The performance measures of interest are throughput efficiency and fairness.

\subsection{Variable Number of Hops Experiments}

We start by examining the interplay of TCP and MAC layers when there is only one TCP connection present in the network. More precisely, we consider a single TCP connection that covers a variable number of hops, from 1 to 5, as depicted in Fig. 1. Here, the distance between neighbor nodes is equal to the radio transmission range. In the experiment, TCP window (W) varies from 1,2, 4, 8 and 16 packets, with each packet being 1460B. The results for CSMA, FAMA and 802.11 throughputs as a function of number of hops $(\mathrm{H})$ and window sizes $(\mathrm{W})$ are reported in Fig. 5a, 5b and $5 \mathrm{c}$. With $\mathrm{W}=1$ packet, one can verify that throughput values match exactly the analytic predictions for a send-and-wait protocol. The throughput is inversely proportional to the hop distance. CSMA throughput is slightly higher than FAMA because of RTS/CTS overhead in the latter. 802.11 bestows the lowest throughput among the three protocols due to the additional ACK required for each data frame and the collision avoidance scheme. 


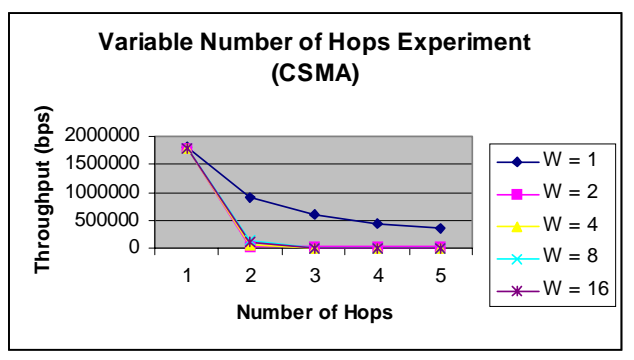

(a) CSMA

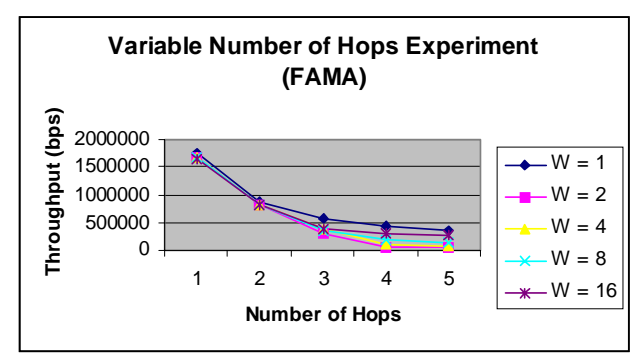

(b) FAMA

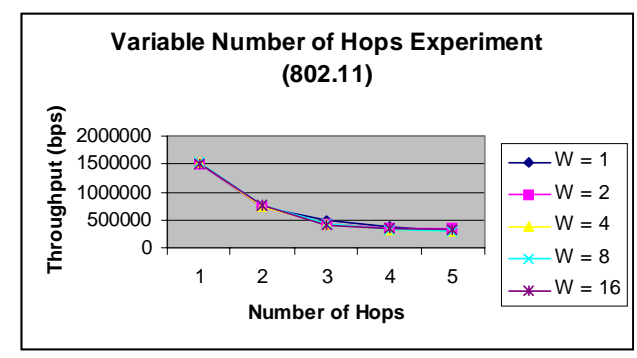

(c) 802.11

Fig. 5: Single TCP Connection, Variable Number of Hops Experiments

As we increase the TCP window (up to 16KB), multiple packets and multiple ACKs travel on the path in the opposite direction, creating interference and collisions. We would expect that in balance the window increase improves performance since for 5 hops, for example, analysis shows that the optimal throughput (assuming optimal scheduling of packet and ACK transmissions along the path) is achieved for $\mathrm{W}=3 \times 1460 \mathrm{~B}$. The simulation results in Fig. 5a indicate that this is not true for CSMA. CSMA throughput collapses when $\mathrm{H}>2$. Hidden terminal losses, which become very substantial for longer paths, cause the loss of TCP ACKs with consequent timeouts and major throughput degradation. FAMA, with a less aggressive yield time, renders better performance compared to that in [9] where FAMA throughput dramatically degrades with $\mathrm{H}>3$. This can be attributed to the fact that with a less aggressive yield time scheme, the collision between data packets and ACKs dramatically decreases due to the fact that the sender yields for an amount of time that is sufficient for the receiver to respond with ACKs. The performance of 802.11 is comparable to that of FAMA. In addition, the throughput of TCP over 802.11 is consistently comparable to that of $\mathrm{W}=1$ packet. Note that the throughput tends to become constant as hop distances grow larger - a typical sign of effective pipelining. Moreover, the asymptotic value of $300 \mathrm{Kbps}$ is not too far off from the theoretical maximum achievable on a multi-hop wireless path, which is $25 \%$ of channel capacity (in our case $0.25 \times 2000 \mathrm{Kbps}=500 \mathrm{Kbps}$ ). The steady performance of 802.11 shown in Fig. $5 \mathrm{c}$ is attributed to the fact that 802.11 acknowledges every frame sent and performs local retransmissions if an ACK for a frame is not received. This precludes TCP ACK loss and consequent TCP timeouts.

From the above results we conclude that it is counterproductive to use $\mathrm{W}$ larger than single packet size even on connections covering multiple hops due to multiple data packets and multiple ACKs colliding on the opposite path. Therefore, we only consider $\mathrm{W}=1460 \mathrm{~B}$ for the remainder of this paper. 


\subsection{Hidden Terminal Experiments}

We next divert our attention to the hidden terminal environment depicted in Fig. 2. Node 1 is in radio range of node 0 and node 2 . Node 0 and node 2 cannot are not within reception range of each other. A single TCP connection is set up from node 0 to node 1 and from node 2 to node 1 . The result of the simulation experiment is shown in Fig. 6.

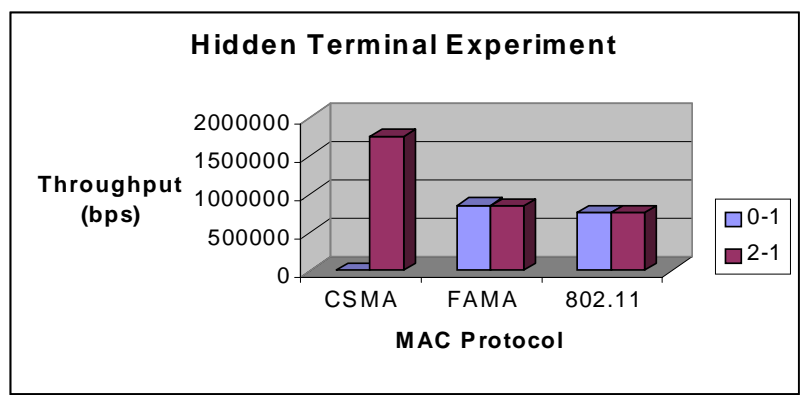

Fig. 6: Throughput (bps) in Hidden Terminal Experiments, $\mathrm{W}=1460 \mathrm{~B}$

As expected, CSMA exhibits capture behavior since both senders are unaware of each other's presence. The connection from node 2 to node 1 monopolizes the channel, with the node 0 to node 1 connection not being able to establish a connection at all. The capture behavior is explained by the interplay of CSMA timeouts with TCP timeouts, coupled with the presence of undetected link losses. When two connections compete for the same channel and one is "pushed back" by the timeouts, the binary exponential backoff nature of both CSMA and TCP timeouts make the situation progressively worse for the loser. FAMA and 802.11, on the other hand, are consistently fair. With FAMA and 802.11, both connections get equal share of the bandwidth with 802.11 attaining aggregate throughput slightly lower than that of FAMA, again due to the additional control frame and collision avoidance scheme. The RTS/CTS mechanism and the less aggressive yield time of FAMA play an important role in the hidden terminal scenario. The RTS/CTS allows node 0 and node 2 to coordinate with one another although they are not within range of each other. This permits the idle source to determine when the other is expected to finish utilizing the wireless medium. The yield time deters the source from capturing the channel by permitting the competing source to transmit on the contention free wireless channel. Likewise, 802.11 succeed in attaining fairness through the RTS/CTS and collision avoidance mechanism. Collision avoidance allows neighboring nodes to coordinate and share the channel. Aggregate throughputs of CSMA (1.8Mbps), FAMA (1.7Mbps) and 802.11 (1.5Mbps) are quite good since the maximum theoretical throughput in a hidden terminal situation is $2 \mathrm{Mbps}$ (only one node can transmit at a time). CSMA and FAMA demonstrates slightly lower aggregate throughput than in the one-hop case reported in Fig. 5a and 5b since useful bandwidth is wasted with packet collisions, causing TCP to timeout and retransmit. 802.11, however, improves on throughput compared to that in the one-hop situation depicted in Fig. 5c. The slight improvement can be attributed to the link level ACK feature of 802.11, thus avoiding TCP timeouts in most cases when packets collide at node 1 .

We note here a phenomenon quite common in multi-access networks, and more generally in resource sharing systems. In order to obtain maximum total throughput, it is often better to select and serve only a few users, so that the control and probing overhead required for fair sharing is eliminated. Capture is an example of this principle. Thus, it is no surprise that the higher the capture in the system, the better the total throughput the system achieves. 


\subsection{Ring Experiments}

The performance of TCP over FAMA and 802.11 seems quite fair with the hidden terminal experiment. To further analyze the interaction of several TCP connections, we consider a ring topology as depicted in Fig. 3. The 8 nodes are engaged in single hop file transfer connections (node 0 to node 1 , node 1 to node $2, \ldots$, node 7 to node 0 ). The results of CSMA, FAMA and 802.11 are illustrated in Fig. 7.

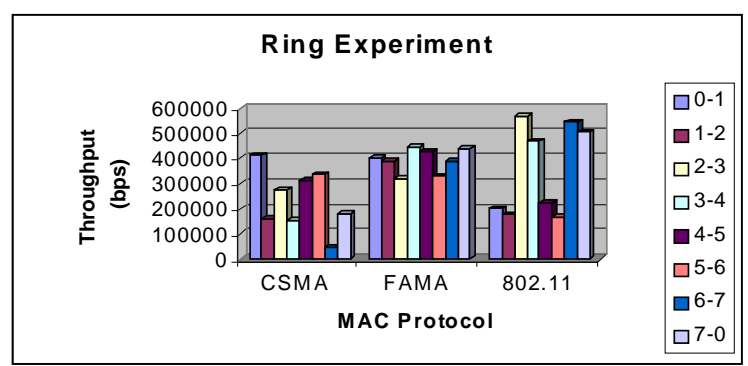

Fig. 7: Throughput (bps), Ring Experiments, $\mathrm{W}=1460 \mathrm{~B}$

We start with CSMA and note that the behavior reveals some level of capture. The connection from node 0 to node 1 obtains almost ten times the throughput $(411 \mathrm{Kbps})$ as that of the connection between node 6 to node 7 (46Kbps). The fairness characteristic is preserved in FAMA, with throughputs ranging from $300 \mathrm{Kbps}$ to $400 \mathrm{Kbps}$. This result is in conflict with the result presented in [9] where FAMA demonstrated major capture effects. The discrepancy is explained by the fact that, in this paper, FAMA possesses a fairer yield time. The added yield time permits neighbors of the sender to equally acquire the channel once the sender transmits a packet, thus leading for fairness. Surprisingly though, 802.11 does not live up to expectation from the hidden terminal experiment. Throughput of 802.11 fluctuates from $167 \mathrm{Kbps}$ to $566 \mathrm{Kbps}$. We note that the sessions from node 2 to node 3 and from node 3 to node 4 achieve similar throughputs while the throughputs of their neighboring connections (node 1 to node 2, node 4 to node 5) are significantly lower. We witness the same phenomenon with the connections from node 6 to node 7 and from node 7 to node 0 . However, by introducing a larger yield time for 802.11 instead of the regular DIFS period, fairness is achieved. Fig. 8 reports the performance of TCP over 802.11 with a larger yield time.

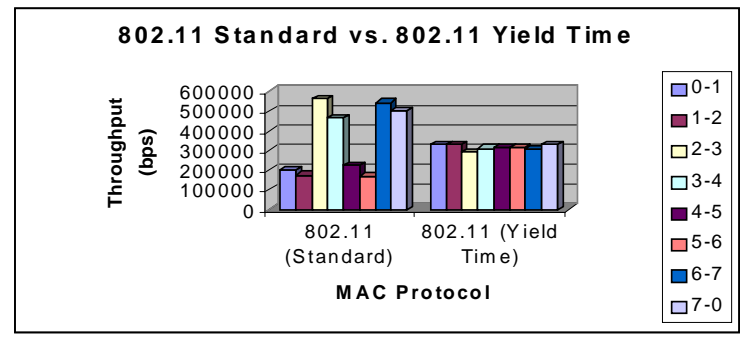

Fig. 8: Throughput (bps), Ring Experiments, 802.11 (Standard) vs. 802.11 (Yield Time),

$$
\mathrm{W}=1460 \mathrm{~B}
$$

Fig. 8 leads us to conclude that MAC layer timers are of great importance in terms of achieving equal throughput among node neighbors and needs to be studied in greater detail to understand its impact on network performance. In terms of overall throughput of all nodes, with FAMA, TCP 
yields aggregate throughput of over $3 \mathrm{Mbps}$. With 802.11 , TCP obtained total throughput of almost $3 \mathrm{Mbps}$ without the introduction of a longer yield time. This compares favorably to the theoretical maximum of $4 \mathrm{Mbps}$. With the introduction of a longer yield time, 802.11 offers total throughput of 2.5Mbps. Thus, 802.11 accomplishes fairness at the cost of total throughput, a familiar tradeoff discussed earlier.

\section{$3.49 \times 9$ Grid Experiments}

In this section we consider a more realistic ad hoc network environment with larger number of nodes and the inclusion of mobility. To this end, we have selected an 81-node grid topology as shown in Fig. 4. The dimension of the grid is 100 X 100 meters. Each node is 10 meters apart from its horizontal and vertical neighbors. Furthermore, each node has a radio power range of 30 meters. Nodes move at a rate of 10 meters per second in a random direction with a probability of 0.5 . When mobility is not considered, static routing is used. When mobility is introduced, Bellman-Ford routing is utilized with routing table updates occurring once every second. FTP connections are established between node 18 to node 26 , node 36 to node 44 , node 54 to node 62 , node 2 to node 74 , node 4 to node 76 and node 6 to node 78. Simulation runs with no mobility are executed for 400 simulated seconds. With mobility, simulation runs last for 800 seconds in simulation time with FTP session starting after 400 seconds. We start with the analysis of the experiments with no mobility (Fig. 9).

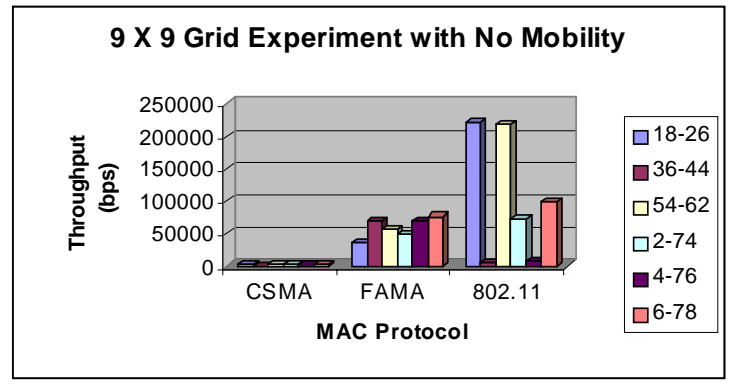

Fig. 9: Throughput (bps), 9 X 9 Grid Experiments, No Mobility, W = 1460B

First, we consider CSMA. Theoretical maximum throughput for an 8-hop connection with W equal to one packet size is 300Kbps. TCP performance under CSMA virtually collapses with maximum throughput of $2.9 \mathrm{Kbps}$ from the connection between node 2 to node 74 . The average throughput is $1.6 \mathrm{Kbps}$. The horrific performance is due to interference from neighbor FTP connections as well as interference from the three cross traffic connections. Particularly damaging to multi-hop FTP connections in CSMA is the high loss rate on the links and the lack of link loss recovery. Next, we shift our attention to FAMA. FAMA throughput is much higher than CSMA, with average throughput of 59.7Kbps. The improvement of FAMA is credited to the RTS/CTS control frames and the fair yield time. Therefore, FAMA is better protected against hidden terminal losses. Minimum and maximum throughput is $36.6 \mathrm{Kpbs}$ and $76.4 \mathrm{Kbps}$, respectively. Compare to the theoretical maximum of $300 \mathrm{Kbps}$, FAMA is still inadequate. With 802.11 , the average throughput is $104 \mathrm{Kbps}$. Minimum and maximum are $7.5 \mathrm{Kbps}$ and $222.3 \mathrm{Kbps}$, respectively, which measure favorably to the theoretical maximum. The improved throughput is the direct result of the link level ACKs, which help achieve good aggregate throughput in a high loss rate environment. However, capture is not alleviated. We next turn our attention to the same configuration except now nodes move at a rate of 10 meters per second in a random fashion with a probability of 0.5 . 


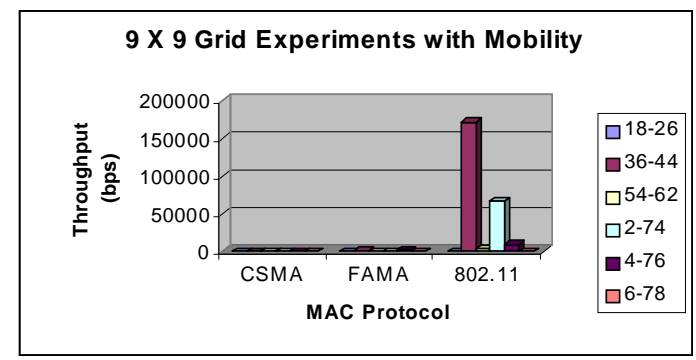

Fig. 10: Throughput (bps), 9 x 9 Grid Experiments, Mobility, $\mathrm{W}=1460 \mathrm{~B}$

From Fig. 10, CSMA collapses with five out of six sources not being able to establish connection. Furthermore, the lone connection that is able to be established (node 36 to node 44) obtained negligible throughput (381 bps). FAMA improves on CSMA, although not by much. Two connections did not get established and another closed due to too many retransmissions by TCP after the successful connection setup phase. 802.11 performs the best out of the three MAC protocols being studied. Still, two of the sources fail to open the TCP session. TCP sessions from node 36 to 44 and from node 2 to node 74 obtain throughputs of $172.2 \mathrm{Kbps}$ and $67 \mathrm{Kpbs}$, respectively, but the rest show only marginal gain.

In summary, the grid experiments confirm the behavior observed in linear topologies. Namely, poor multi-hop connection performance exists under TCP, especially in conjunction with CSMA. FAMA performs better than CSMA, both in absence and in presence of mobility. It is clear, however, that neither FAMA nor CSMA are adequate for ad hoc network operation without the assistance of link level loss protection and of proper fairness measures. 802.11 is by far superior to both CSMA and FAMA in terms of throughput. However, 802.11 shows evidence of capture effects. When mobility is introduced, CSMA and FAMA collapse. Only 802.11 demonstrates signs of productivity.

\section{Conclusion}

The focus of this paper has been the TCP/MAC interaction in a multi-hop radio network. In particular, we focus on TCP performance over CSMA, FAMA and 802.11 and examine the fair sharing of bottlenecks. We first note that the best performance is achieved with $\mathrm{W}=1$ packet. With an adaptive window, conflicts between multiple packets and outstanding ACKs arise. We observed capture in 802.11 under the ring experiment but notice that by adjusting the yield time of 802.11, capture is eliminated (at the expense of reduction in aggregate throughput). Finally, we show that, in the face of mobility, MAC protocols without link loss protection break down. Between the three MAC layer protocols under study, CSMA performs best only when there are no competing TCP streams. FAMA works well in most situations, although it collapses when mobility is introduced. 802.11 shows the most promise, giving a good combination of aggregate throughput and fairness.

The results indicate that further research is necessary to make TCP and MAC layers work consistently well together in a multi-hop environment. More work needs to be done on MAC layer timers and on their interplay with upper layer backoff schemes. Queue scheduling within the wireless node is another area that requires more research. For example, will fair queueing provide fairness in bottleneck links spanning multiple hops? Also, the interplay between backoff policy, link protection scheme and floor acquisition methods must be investigated in greater detail. For example, which of 
the above features is really responsible for improving performance in any of the situations considered in this study? Finally, more work must be done regarding MAC layer support for mobility.

\section{Acknowledgements}

This research was supported in part by DARPA under contract DAAB07-97-C-D321, by NSF under contract ANI-9814675, by Intel under project "QoS Wireless Networks".

\section{References}

[1] R. Bagrodia, R. Meyer, et al., "PARSEC: A Parallel Simulation Environment for Complex System", Computer Magazine, 1998.

[2] R. Bagrodia and M. Gerla, "A Modular and Scalable Simulation Tool for Large Wireless Networks", International Conference on Modeling Techniques and Tools for Computer Performance Evaluation, 1998.

[3] Bikram Bakshi, Krishna, P., Pradhan, D.K., and Vaidya, N.H., "Performance of TCP over Wireless Networks", 17th Intl. Conf. on Distributed Computing Systems (ICDCS), Baltimore, May 1997.

[4] Hari Balakrishnan, Srinivasan Seshan, Randy H. Katz, "Improving Reliable Transport and Handoff Performance in Cellular Wireless Networks", ACM Wireless Networks, 1(4), December 1995.

[5] V. Bharghavan, A. Demers, S. Shenker, and L. Zhang, "MACAW: A Media Access Protocol for Wireless LAN's," ACM SIGCOMM, 1994.

[6] Editors of IEEE 802.11, Wireless LAN Medium Access Control (MAC and Physical Layer (PHY) specifications, Draft Standard IEEE 802.11, P802.11/D5.0, July 1996.

[7] C. Fullmer and J.J. Garcia-Luna-Aceves, "Floor Acquisition Multiple Access (FAMA) for packet radio networks", Computer Communication Review, vol. 25, (no. 4), (ACM SIGCOMM '95, Cambridge, MA, USA, 28 Aug.-1 Sept. 1995.) ACM, Oct. 1995.

[8] M. Gerla and J.T.-C. Tsai, "Multicluster, mobile, multimedia radio network", ACM/Baltzer Journal of Wireless Networks. vol. 1, (no. 3), 1995, p. 255-265.

[9] Mario Gerla, Ken Tang, Rajive Bagrodia, "TCP Performance in Wireless Multihop Networks", Proceedings of IEEE WMCSA'99, Feb. 1999.

[10] John Jubin and Janet D. Tornow, "The DARPA Packet Radio Network Protocols", Proceedings of the IEEE, Jan. 1987.

[11] Andrew S. Tanenbaum, "Computer Networks: Third Edition”, Prentice Hall PTR, New Jersey, 1996.

[12] X. Zeng, R. Bagrodia and M. Gerla, "GloMoSim: a Library for the Parallel Simulation of Largescale Wireless Networks", PADS, 1998.

[13] M. A. Marsan and Mario Gerla, "Fairness in Local Computer Networks", ICC Conference Proceedings, June 1982. 